\title{
Parameters of the Three-Pool Model of the Turnover of Plasma Cholesterol in Normal and Hyperlipidemic Humans
}

\author{
Frank Rees Smith, Ralph B. Dell, Robert P. Noble, and DeWitt S. Goodman \\ From the Departments of Medicine and Pediatrics, Columbia University College \\ of Physicians and Surgeons, New York, 10032, and The Sharon Research \\ Institute, Sharon Hospital, Sharon, Connecticut 06069
}

A B S T R A C T Long-term studies (32-49 wk) of the turnover of plasma cholesterol were conducted in 24 subjects. Eight subjects were normolipidemic, six had hypercholesterolemia, eight had hypercholesterolemia and hypertriglyceridemia, and two had hypertriglyceridemia alone. 10 of the hyperlipidemic patients had a definite familial disorder. In all subjects (except one for whom complete data were not available), the same three-pool model previously described gave the best fit for the data. The parameters of the three-pool model observed in the normal subjects were compared with the model parameters found in the patients with the different kinds of hyperlipidemia. In addition, single and multiple regression analyses were conducted to explore the relationships between the model parameters and various physiological variables, including age, body size, and serum lipid concentrations.

Using this approach, significant differences between groups, or correlations with serum lipid levels were seen for several parameters of the three-pool model: the production rate $(P R)$; the size of the rapidly exchanging pool $1\left(M_{1}\right)$; all estimates of the size of the most slowly equilibrating pool $3\left(\mathrm{M}_{3}\right)$; and the rate constant $k_{\text {21. }}$. The $\mathrm{PR}$ in normal subjects $(1.14 \pm 0.19 \mathrm{~g} /$ day, mean $\pm \mathrm{SD}$ ) was not significantly different from that found in patients with hypercholesterolemia, with or without hypertriglyceridemia. The major determinant of cholesterol PR was overall body size, expressed either as total body weight or as surface area. The correlations between $\mathrm{PR}$ and indices of adiposity (percent ideal weight and excess weight), although statistically significant, were much weaker in this nonobese population. After adjustment for body size variation, cholesterol PR was not correlated with the serum cholesterol con-

Dr. Dell is a Career Scientist of the Health Research Council of the City of New York.

Received for publication 16 May 1975 and in revised form 29 August 1975. centration but was probably $(P<0.05)$ correlated with the triglyceride concentration. When the two patients with very high triglyceride concentrations were excluded, however, no correlation was observed between adjusted PR and triglyceride level. It is probable that hypertriglyceridemic patients represent a heterogeneous population, in which the majority do not show increased cholesterol PR.

$M_{1}$ was correlated with all body size variables, but most strongly with excess weight. After adjusting for the effects of body size, $M_{1}$ was also correlated with the serum concentrations of both cholesterol and triglyceride. Major differences were found in the relationships between the physiological variables and the sizes of pools 2 and $3 . M_{2}$ was correlated neither with any of the indices of body size or adiposity, nor with the serum levels of either cholesterol or triglyceride. In contrast, all estimates of $\mathrm{M}_{3}$ were correlated with indices of adiposity (but not of overall body size) and with the serum cholesterol concentration. Thus, the amount of cholesterol in slowly equilibrating tissue sites appears to particularly increase with elevations of the serum cholesterol level. The results also confirm previous data that adipose tissue cholesterol is an important part of pool 3.

\section{INTRODUCTION}

The turnover of plasma cholesterol has been studied in recent years to provide information about the metabolism of cholesterol in normal subjects and in patients with hyperlipidemia and with obesity (1-7). In 1968 two of us reported (1) that the plasma cholesterol specific radioactivity-time curves obtained in experiments of about 10 wk duration could be resolved into two exponential functions and, hence, that the turnover of plasma cholesterol conformed to a simple two-pool model. In 1970, Samuel and Perl (5) reported that in 
some patients the slow slope of the plasma decay curve deviated from monoexponential behavior after approximately 20-25 wk. This suggested that a multicompartmental model of more than two pools was necessary to describe the long-term turnover of plasma cholesterol in man. The turnover of plasma cholesterol was subsequently studied by us for periods of $32-41$ wk in six subjects and, in each subject, the best description of the turnover curve was found to be provided by a three-pool rather than a two-pool model (6).

We now report the results of long-term studies of the turnover of plasma cholesterol in 24 subjects. The parameters of the three-pool model of cholesterol turnover observed in eight normal subjects have been compared with the parameters found in six patients with hypercholesterolemia and in eight patients with both hypercholesterolemia and hypertriglyceridemia (mixed hyperlipidemia). In addition, relationships between the model parameters of cholesterol turnover and the physiological variables of age, body size, serum cholesterol, and serum triglyceride concentrations have been explored, using simple linear correlations and multiple linear regression analyses.

\section{METHODS}

24 volunteer subjects participated in these studies. Written informed consent was obtained from each. The characteristics of the subjects studied are shown in Table I. Eight subjects were normal controls; six had elevated serum cholesterol concentrations with normal triglyceride concentrations; eight had elevations of both serum cholesterol and triglyceride concentrations; and two had elevations of serum triglyceride concentrations alone. The diagnosis of hyperlipidemia was based on an arbitrary working definition of levels of cholesterol (at the time of the study) in excess of $275 \mathrm{mg} / \mathrm{dl}$, and/or levels of triglyceride in excess of $160 \mathrm{mg} / \mathrm{dl}$ in serum obtained after a 12-h fast. In analyzing the data, patients were classified as having hypercholesterolemia, hypertriglyceridemia, or both (mixed hyperlipidemia).

Table I also shows the lipoprotein phenotype $(8,9)$ as determined by the lipoprotein pattern seen after electrophoresis in agarose gel (10). The patients with hypercholesterolemia or hypertriglyceridemia alone were judged to have the type IIa or type IV patterns, respectively. Seven of the eight patients with mixed hyperlipidemia had the type IIb pattern. One patient, D. F., had a "broad beta band" on electrophoresis; the diagnosis of type III was confirmed by ultracentrifugation $(8,9)$. Four of the hypercholesterolemic patients had clinical ischemic heart disease, as indicated by previously documented myocardial infarctions (J. Z., R. K., and J. R., at ages 45,55 , and $32 \mathrm{yr}$, respectively), or by the presence of angina pectoris (A. A.).

A genetic classification of each hyperlipidemic patient was attempted, following the approach of Goldstein et al. (11). Only first-degree relatives were used in the genetic analysis. Cholesterol and triglyceride concentrations were measured, in sera obtained after a 12-h fast, in 56 (of 69 potentially available) first-degree relatives. The goal, for diagnosis, was to test serum from as many as possible, but with a minimum of three first-degree relatives in each kindred. To make a positive designation of familial disease (familial disease present), one relative was required to have serum cholesterol or triglyceride level greater than or equal to the 99th percentile, or if under $20 \mathrm{yr}$ of age, greater than the 95th percentile value. The regression equations reported by Goldstein et al. $(11,12)$ were used to correct cholesterol and triglyceride concentrations in members of the kindreds for age. The 95th and 99th percentile cutoff values established in the Seattle study (12) were used. 10 of the hyperlipidemic subjects were found to have familial disease by this criterion. For 7 of these 10 subjects, 3 or more first-degree relatives were tested; only 2 first-degree relatives were tested for each of the other 3 hyperlipidemic subjects with familial disease (see Table I). Familial disease was considered to be absent if at least three firstdegree relatives in the kindred were tested, and no relative had hyperlipidemia as defined above. None of the three hyperlipidemic subjects in whom familial disease was absent (see Table I) had a tested first-degree relative with a lipid level greater than the 95th percentile cutoff value. If less than three first-degree relatives were available or were tested in a kindred, and if unequivocal hyperlipidemia was not found in those relatives tested, the possible presence of a familial disorder was considered to be "indeterminate" (three hyperlipidemic subjects, see Table I). In several kindreds, where a familial disorder was definitely present, the lipid levels and distribution conformed to the genetic classification of either familial hypercholesterolemia, or of familial "combined hyperlipidemia," as defined by Goldstein et al. (11). If the genetic classification was not clear, it was labeled as "not definite" (see Table I).

All subjects were studied as outpatients. Before the study, all patients had been instructed in a diet containing less than $300 \mathrm{mg}$ of cholesterol per day with less than $35 \%$ of calories as total fat and less than $10 \%$ as saturated fat. All patients had been stabilized on the diet for at least several weeks before the study was begun, and were specifically asked not to modify their diets during the course of the study. The normal control subjects ate their usual diets. None of the subjects had significant weight change during the study. Serum cholesterol and triglyceride concentrations were reasonably stable during the study (note the small standard error values for lipid levels in Table I).

$\left[4-{ }^{14} \mathrm{C}\right]$ Cholesterol (specific radioactivity $58 \mu \mathrm{Ci} / \mu \mathrm{mol}$, New England Nuclear, Boston, Mass.) complexed with serum lipoprotein was injected intravenously, and the specific radioactivity of serum total cholesterol was determined on samples collected serially thereafter. The methods used have been described in detail previously $(1,6)$. In each experiment the $\left[{ }^{14} \mathrm{C}\right]$ cholesterol was checked for radiopurity, by thin-layer chromatography, just before use. The amounts of radioactivity injected (from 22 to $25 \mu \mathrm{Ci}$ per subject) were measured precisely, and the data were later adjusted to an injected dose of $25 \mu \mathrm{Ci}$ for each subject. Samples of venous blood were collected before breakfast on days 1 , $3,6,8,10$, and 14 after injection, and thereafter at frequencies decreasing from every week to every 3 wk for a total of 34-40 samples during study periods of from 32 to 49 wk. The specific radioactivity of the cholesterol in each sample was determined by analysis of the nonsaponifiable lipid extract obtained from the sample as described in detail previously (6). The serum was also used for measurement of the concentrations of cholesterol and triglyceride with a Technicon AutoAnalyzer I (Technicon Instruments Corp., Tarrytown, N. Y.) using the method N-24a for cholesterol (13), and a modification (14) of the Kessler and Lederer technique (15) for triglycerides. 
TABLE I

Characteristics of Subjects Studied

\begin{tabular}{|c|c|c|c|c|c|c|c|c|c|c|c|c|c|}
\hline \multirow[b]{2}{*}{ Subject } & \multirow[b]{2}{*}{$\begin{array}{l}\text { Study } \\
\text { duration }\end{array}$} & \multirow[b]{2}{*}{ Sex } & \multirow[b]{2}{*}{ Age } & \multirow[b]{2}{*}{ Height } & \multirow[b]{2}{*}{ Weight } & \multirow{2}{*}{$\begin{array}{c}\text { Ideal } \\
\text { body } \\
\text { wt } \\
(18)\end{array}$} & \multicolumn{2}{|c|}{ Serum } & \multirow[b]{2}{*}{$\begin{array}{l}\text { Classifi- } \\
\text { cation } \ddagger\end{array}$} & \multirow[b]{2}{*}{$\begin{array}{l}\text { Xan- } \\
\text { tho- } \\
\text { mata\& }\end{array}$} & \multirow[b]{2}{*}{$\begin{array}{c}\text { LP } \\
\text { pattern } \\
\text { (type) }\end{array}$} & \multirow[b]{2}{*}{$\begin{array}{c}\text { Familial } \\
\text { disorder } 8\end{array}$} & \multirow[b]{2}{*}{$\begin{array}{l}\text { Genetic } \\
\text { classifi- } \\
\text { cation \| }\end{array}$} \\
\hline & & & & & & & $\begin{array}{l}\text { Choles- } \\
\text { terol* }\end{array}$ & $\begin{array}{l}\text { Triglyc- } \\
\text { eride* }\end{array}$ & & & & & \\
\hline & $w k$ & & $y r$ & $\mathrm{~cm}$ & $\mathrm{~kg}$ & $\%$ & \multicolumn{2}{|c|}{$m g / d l$} & & & & & \\
\hline F. C. श & 41 & $\mathbf{M}$ & 51 & 175 & 76.2 & 112 & $220 \pm 2$ & $85 \pm 4$ & NL & - & NL & & \\
\hline R. M. & 32 & $\mathbf{M}$ & 53 & 183 & 76.2 & 102 & $231 \pm 3$ & $102 \pm 3$ & NL & - & NL & & \\
\hline R. N. I & 39 & $\mathbf{M}$ & 54 & 183 & 79.4 & 108 & $211 \pm 2$ & $69 \pm 3$ & $\mathrm{NL}$ & - & NL & & \\
\hline B. B. & 46 & $\mathbf{M}$ & 23 & 183 & 75.0 & 99 & $178 \pm 2$ & $110 \pm 5$ & NL & - & NL & & \\
\hline R. F. & 46 & $\mathbf{M}$ & 24 & 187 & 81.4 & 101 & $212 \pm 3$ & $112 \pm 4$ & NL & - & NL & & \\
\hline R. H. & 46 & $\mathbf{M}$ & 29 & 179 & 73.7 & 101 & $167 \pm 2$ & $91 \pm 3$ & $\mathrm{NL}$ & - & NL & & \\
\hline A. $\mathrm{R}$. & 35 & $\mathbf{M}$ & 37 & 170 & 68.2 & 100 & $208 \pm 3$ & $137 \pm 7$ & NL & - & NL & & \\
\hline G. W. & 37 & $\mathbf{M}$ & 44 & 187 & 79.5 & 99 & $203 \pm 2$ & $106 \pm 6$ & NL & - & NL & & \\
\hline J. Z. & 40 & $\mathbf{M}$ & 56 & 165 & 63.2 & 98 & $480 \pm 6$ & $93 \pm 2$ & H-Chol & - & I Ia & + & $\mathrm{FH}$ \\
\hline V. M. & 42 & $F$ & 62 & 156 & 52.7 & 100 & $280 \pm 3$ & $103 \pm 4$ & H-Chol & - & IIa & + & ND \\
\hline R. K. & 38 & $F$ & 57 & 152 & 50.0 & 99 & $420 \pm 4$ & $157 \pm 8$ & H-Chol & - & $\mathrm{IIa}$ & - & \\
\hline A. A. & 40 & $F$ & 74 & 162 & 66.8 & 118 & $484 \pm 4$ & $127 \pm 5$ & H-Chol & + & IIa & IND & \\
\hline A. N. & 49 & $\mathbf{M}$ & 44 & 183 & 102.3 & 124 & $428 \pm 5$ & $135 \pm 8$ & H-Chol & - & I Ia & + & FH \\
\hline J. R. & 46 & $\mathbf{M}$ & 34 & 170 & 64.1 & 100 & $277 \pm 3$ & $104 \pm 3$ & H-Chol & - & IIa & - & \\
\hline E. M. & 42 & $\mathbf{M}$ & 52 & 175 & 62.7 & 93 & $283 \pm 7$ & $162 \pm 9$ & Mixed & - & IIb & + & Comb \\
\hline C. S. & 40 & $\mathbf{M}$ & 52 & 185 & 85.9 & 101 & $318 \pm 4$ & $233 \pm 12$ & Mixed & - & IIb & $-* *$ & \\
\hline J. P. & 41 & $\mathrm{~F}$ & 54 & 165 & 56.4 & 96 & $551 \pm 7$ & $230 \pm 8$ & Mixed & + & IIb & $+\ddagger$ & $\mathrm{FH}$ \\
\hline N. D. & 41 & $\mathbf{M}$ & 53 & 173 & 86.4 & 117 & $335 \pm 3$ & $177 \pm 7$ & Mixed & - & IIb & $+\ddagger t$ & ND \\
\hline D. F. & 46 & $\mathbf{M}$ & 41 & 173 & 75.5 & 106 & $418 \pm 11$ & $691 \pm 35$ & Mixed & + & III & + & ND \\
\hline J. F. & 46 & $\mathbf{M}$ & 47 & 171 & 84.1 & 113 & $424 \pm 8$ & $464 \pm 20$ & Mixed & + & IIb & + & ND\&8 \\
\hline C. G. & 49 & $\mathrm{~F}$ & 67 & 173 & 60.0 & 94 & $345 \pm 5$ & $215 \pm 7$ & Mixed & - & IIb & + & Comb \\
\hline H. L. I & 37 & $\mathbf{M}$ & 40 & 173 & 79.4 & 115 & $295 \pm 5$ & $280 \pm 24$ & Mixed & - & IIb & $+\ddagger$ & ND \\
\hline G. F. I & 41 & $\mathbf{M}$ & 54 & 180 & 73.9 & 104 & $240 \pm 2$ & $216 \pm 11$ & H-TG & - & IV & IND & \\
\hline J. B. I & 36 & $\mathbf{M}$ & 61 & 145 & 63.5 & 100 & $223 \pm 3$ & $294 \pm 13$ & H-TG & - & IV & IND & \\
\hline
\end{tabular}

* Mean \pm SEM during the period of the study (34-40 samples per subject).

† Classifications: NL, normal; H-Chol, hypercholesterolemia; H-TG, hypertriglyceridemia; Mixed, mixed hyperlipidemia (see text).

$\delta(+)=$ present $;(-)=$ absent $;$ IND = indeterminate.

|| Classifications: FH, familial hypercholesterolemia; Comb, familial combined hyperlipidemia; ND, not definite; No notation is made in this column for subjects where a familial disorder was absent or was "indeterminate."

I Subject previously reported (6).

** Although three first-degree relatives were tested and were all normolipidemic by history, three brothers of this patient had died in their fifth decade of myocardial infarction.

\# Only two first-degree relatives tested. For J. P., both relatives (mother and daughter) had hypercholesterolemia. For N. D., one relative (son, age $18 \mathrm{yr}$ ) had hypercholesterolemia (306) and hypertriglyceridemia (257), and one relative (sister) was normal. For H. L., both relatives (one brother and one sister) had hypertriglyceridemia, and both had acute myocardial infarctions in the mid-50's (age 56 and $55 \mathrm{yr}$, respectively).

$\$ \$ 6$ of 11 first-degree relatives tested had hypertriglyceridemia (type IV pattern).

The data for each subject were analyzed by digital computer, using a weighted, least-squares, nonlinear regression technique as described elsewhere $(6,16)$, to determine the parameters of a three-pool mammillary model which would provide the best fit. The model used is illustrated in Fig. 1. The notation is identical with that described previously (6). The pools are denoted by arabic numbers (pools 1, 2, and $3)$; rate constants are noted by $k ;^{1}$ the rate of transfer of

${ }_{1}^{1}$ Abbreviations used in this paper: $k_{12}, k_{21}, k_{13}, k_{31}$, rate constants; LDL, low density lipoprotein; $\mathbf{M}_{\mathbf{1}}, \mathbf{M}_{\mathbf{2}}, \mathbf{M}_{\mathbf{3}}$, pool cholesterol mass (in grams/day) into or out of a pool is denoted by $R$; cholesterol production rate (PR) is given by $R_{01}$; pool sizes are designated $\mathrm{M}_{1}, \mathrm{M}_{2}$, and $\mathrm{M}_{\mathbf{3}}$. For this model, the equation for the specific activity of pool 1 is:

$$
\text { sp act }=A_{1} e^{-\alpha_{1} t}+A_{2} e^{-\alpha_{2} t}+A_{3} e^{-\alpha_{2} t} \text {. }
$$

Equations relating the constants $(A$ and $\alpha$ ) in this equation to the model parameters have been reported (6).

sizes; $\mathrm{PR}$, production rate; $R$, rate of transfer of cholesterol mass. 


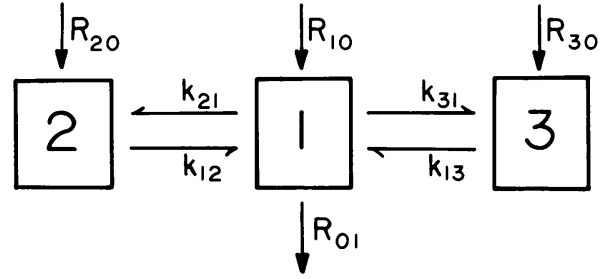

Figure 1 Three-pool model of cholesterol turnover in man (see text for definition and discussion of symbols).

Analysis of the turnover data in terms of this three-pool model provides unique values for each of the rate constants for transfer between pools $\left(k_{21}, k_{12}, k_{31}\right.$, and $\left.k_{13}\right)$; for $\mathrm{PR}$; and for $M_{1}$ (the size of the rapidly turning over pool). Although unique values cannot be obtained for the sizes of the more slowly turning over pools 2 and 3, upper and lower limiting values for $\mathbf{M}_{2}$ and $\mathrm{M}_{3}$ can be calculated, as described previously (6), by making assumptions about the amount and distribution of synthesis of cholesterol outside of pool 1. Thus, minimum values of $M_{2}$ and $M_{3}$ are obtained by assuming that all of cholesterol production enters pool 1 (i.e. $\mathrm{PR}=R_{10}$, where $\mathrm{PR}$ represents the sum of endogenously synthesized cholesterol plus absorbed cholesterol which is derived from the diet). Upper limiting values for $\mathrm{M}_{2}$ or $\mathrm{M}_{3}$ are obtained by assuming that all of cholesterol synthesis (i.e. $P R-0.2$, assuming $R_{10}=0.2 ;[6]$ ) occurs in either pool 2 or 3 , respectively. In addition, in the present study 'intermediate' values of $\mathrm{M}_{2}$ and $\mathrm{M}_{3}$ were calculated by assuming that $R_{10}=0.2$ and that the rest of $\mathrm{PR}$ was evenly divided between $R_{20}$ and $R_{30}$.

The data were analyzed statistically by examining, for each subject, 23 physiologic variables or model parameters. The 23 variables were: age; five variables related to body size : height, weight, surface area (defined as $\mathrm{W}^{0.425} \times \mathrm{H}^{0.725}$ $\times 0.007184(17)$, where $\mathrm{W}$ is body weight in kilograms, $\mathrm{H}$ is height in centimeters, and surface area is given in square meters), percent of ideal body weight (defined as the actual weight divided by the mean desirable weight for the patient's frame as determined from actuarial data [18]), and excess weight (defined as total body weight minus ideal body weight); serum cholesterol and triglyceride concentrations; six uniquely determined model parameters (see above); two transfer rates, $R_{2}$ and $R_{3}$ (defined as $\mathrm{M}_{1} k_{21}$ and $\mathrm{M}_{1} k_{31}$, respectively); and seven estimates of $\mathrm{M}_{2}$ and $\mathrm{M}_{3}$ (minimum, maximum, and intermediate values, plus the sum of intermediate $\mathrm{M}_{2}+\mathrm{M}_{3}$ ).

Initially, the patients were divided into groups according to the kind of lipid elevation present (see Table I), and mean and standard deviation values were calculated for each variable. A one-way analysis of variance was then performed (19) for each of the model parameters. When the variation between groups was found to be significant, the parameter values for each hyperlipidemic group were compared with those of the normal group using the Dunnett procedure for comparing several means with a control mean (19); and the significance of differences at the 5 and $1 \%$ level was assessed using Dunnett's table (reference 19, Table A9, p. 446).

Analyses were next conducted to determine whether there was a simple, linear correlation between any of the model parameters and any of the physiological variables. Based on the results of these correlation analyses, a multiple linear regression analysis was performed using the equation

$$
y=a+\sum_{i=1}^{n} b_{i} x_{i}(20) \text {. }
$$

The dependent variables ( $y$ values) were each of the model parameters in turn. The independent variable $\left(x_{1}\right)$ was first the best body size correlate (from the linear regression analysis described above), and then the serum cholesterol and triglyceride concentrations. The significance of each term $\left(b_{i} x_{\mathfrak{i}}\right)$ in explaining the variation in the dependent variable was assessed by testing the significance of the decrease in the residual error about the regression plane.

\section{RESULTS}

Appropriate model. As described above, the data for each subject were analyzed to determine the parameters which would provide the best fit obtainable with a threepool mammillary model. The data were also analyzed to determine the parameters which would provide the best fit obtainable with a two-pool model, and with models containing more than three pools. Residual error testing was used, as described previously (6), to find the number of pools needed to describe best the specific radioactivity-time curves.

In each of the 23 subjects in whom complete data were available, the three-pool model provided a significantly better fit $(P<0.01$ in 18 subjects and $P$ $<0.05$ in 5 subjects) to the data than did a two-pool model. In one subject ([A. A.] because of a period when she was absent from this country), samples were not collected between weeks 14 and 25 of her 40 wk study. As a consequence, although the parameters of the three-pool model could be estimated satisfactorily in this patient, the fit obtained with this model was not significantly better than that obtained with a twopool model. No further improvement in fit was obtained for any subject with a four-compartment model. Thus, the same three-pool model (Fig. 1) provided the best description of the long-term turnover curves for both the normal controls and the different kinds of hyperlipidemic subjects.

Between group comparisons. The means and standard deviations are presented for each variable and model parameter, by group and for the total population studied, in Tables II-IV. ${ }^{2}$ To test for the presence of significant differences between the groups, a one-way analysis of variance was performed for each physiological variable and each model parameter. For each param-

additional values for the model parameters for each of the 24 long-term studies in Table II have been deposited with Microfiche Publications. See NAPS document 02695 for 1 page of the supplementary material. Order from ASIS/NAPS, c/o Microfiche Publications, 440 Park Avenue South, New York 10016. Remit with order for each NAPS document number $\$ 3.00$ for microfiche and $\$ 5.00$ for photocopies. Make checks payable to Microfiche Publications. 
TABLE II

Subjects Studied by Groups: Physiological Variables (mean $\pm S D$ )

\begin{tabular}{|c|c|c|c|c|c|c|c|c|}
\hline \multirow{2}{*}{$\begin{array}{c}\text { Group } \\
\text { (no. of subjects) }\end{array}$} & \multirow[b]{2}{*}{ Age } & \multirow[b]{2}{*}{ Height } & \multirow[b]{2}{*}{ Weight } & \multirow[b]{2}{*}{ Surface area } & \multirow{2}{*}{$\begin{array}{c}\text { Ideal } \\
\text { body wt }\end{array}$} & \multirow[b]{2}{*}{ Excess weight } & \multicolumn{2}{|c|}{ Serum } \\
\hline & & & & & & & Cholesterol & Triglyceride \\
\hline & $y r$ & $\mathrm{~cm}$ & $\mathrm{~kg}$ & $m^{2}$ & $\%$ & $\mathrm{~kg}$ & \multicolumn{2}{|c|}{$m g / d l$} \\
\hline Normal (8) & $39.4 \pm 13.0$ & $181 \pm 6$ & $76 \pm 4$ & $1.96 \pm 0.09$ & $102 \pm 5$ & $1.94 \pm 3.29$ & $204 \pm 21$ & $101 \pm 20$ \\
\hline Hypercholesterolemia (6) & $54.5 \pm 14.0$ & $165 \pm 11 \ddagger$ & $66 \pm 19$ & $1.73 \pm 0.28$ & $107 \pm 11$ & $4.69 \pm 8.53$ & $395 \pm 94$ & $120 \pm 24$ \\
\hline Mixed hyperlipidemia (8) & $50.8 \pm 8.5$ & $174 \pm 6$ & $74 \pm 12$ & $1.87 \pm 0.16$ & $104 \pm 10$ & $3.35 \pm 6.85$ & $371 \pm 89$ & $306 \pm 182$ \\
\hline Hypertriglyceridemia (2) & $57.5 \pm 5.0$ & $163 \pm 25^{*}$ & $69 \pm 7$ & $1.74 \pm 0.27$ & $102 \pm 3$ & $1.42 \pm 2.01$ & $232 \pm 12$ & $255 \pm 55$ \\
\hline All subjects (24) & $48.5 \pm 12.8$ & $173 \pm 11$ & $72 \pm 12$ & $1.85 \pm 0.20$ & $104 \pm 8$ & $3.05 \pm 5.91$ & $310 \pm 110$ & $187 \pm 140$ \\
\hline
\end{tabular}

Probability that value differs from normal by one-way analysis of variance and use of the Dunnett procedure (see Methods): * $P<0.05 ; \ddagger P<0.01$.

eter, the pooled standard deviation was calculated and the significance of the variation between groups was tested with an $\mathrm{F}$-test. If the overall $\mathrm{F}$ was significant, we tested between group differences by comparing each hyperlipidemic group to the normal group by the Dunnett procedure (see Methods).

In each of the groups studied, the mean value for percent of ideal body weight did not differ significantly from $100 \%$, nor were there significant differences in mean percent of ideal body weight values between groups (Table II). The only significant difference in physiological variables (Table II) (other than in serum lipid levels, which were the criteria on which the groups were classified) was found in height. Thus, the hypercholesterolemic and hypertriglyceridemic groups were both shorter than the normal group. The normal subjects differed from the hyperlipidemic groups in having a lower mean age, but this difference was not significant statistically. Furthermore, the normal controls were all males, whereas three of the six hypercholesterolemic subjects, and two of the eight subjects with mixed hyperlipidemia were females. No differences related to sex were apparent in any of the data, so that this was felt not to represent a confounding variable. In addition, it should be noted that the hyperlipidemic subjects were all on a fat-controlled diet (see Methods), whereas the normal controls ate their usual diets which were somewhat higher in cholesterol and saturated fat content.

For the unique model parameters (Table III), significant variation between groups was found only for $k_{12}$ and $k_{21}$. The PR values for the patients with hypercholesterolemia $(0.98 \pm 0.25)$, and for those with mixed hyperlipidemia $(1.19 \pm 0.28)$, did not differ significantly from the $P R$ of normal control subjects (1.14 \pm 0.19$)$. Although the mean values for $M_{1}$ were higher in the hypercholesterolemic group $(30.9 \pm 8.6)$ and in the mixed hyperlipidemia group (28.4 \pm 4.4$)$ compared with normals $(25.9 \pm 2.8)$, the differences with these fairly small sized groups did not attain statistical significance.

As shown in Table IV, the values for $M_{3}$ (minimum, maximum, and intermediate values) were significantly increased above normal in the mixed hyperlipidemia and hypercholesterolemia groups. In contrast, no difference between groups were seen with any of the estimates of M.

Relationships between model parameters and physiological variables. A simple linear correlation analysis was used to explore relationships between the parameters of the three-pool model and the various physiological variables. The correlations between cholesterol PR and four variables of body size were examined. Two of these variables (total body weight and surface

TABLE III

Subjects Studied by Groups: Unique Model Parameters (mean $\pm S D$ )

\begin{tabular}{|c|c|c|c|c|c|c|c|c|}
\hline Group (no. of subjects) & PR & $\mathbf{M}_{1}$ & $k_{12}$ & $k_{21}$ & $k_{12}$ & $k_{31}$ & $R_{2}$ & $\boldsymbol{R}_{\mathbf{z}}$ \\
\hline & $g / d a y$ & $g$ & $d a y^{-1}$ & $d a y^{-1}$ & $d a y^{-1}$ & $d a y^{-1}$ & B/day & B/day \\
\hline Normal (8) & $1.14 \pm 0.19$ & $25.9 \pm 2.8$ & $0.071 \pm 0.019$ & $0.061 \pm 0.015$ & $0.018 \pm 0.008$ & $0.019 \pm 0.014$ & $1.57 \pm 0.40$ & $0.49 \pm 0.35$ \\
\hline Hypercholesterolemia (6) & $0.98 \pm 0.25$ & $30.9 \pm 8.6$ & $0.079 \pm 0.059$ & $0.037 \pm 0.013^{*}$ & $0.016 \pm 0.005$ & $0.021 \pm 0.011$ & $1.14 \pm 0.59$ & $0.68 \pm 0.42$ \\
\hline Mixed hyperlipidemia (8) & $1.19 \pm 0.28$ & $28.4 \pm 4.4$ & $0.073 \pm 0.037$ & $0.044 \pm 0.014 \S$ & $0.017 \pm 0.007$ & $0.026 \pm 0.014$ & $1.23 \pm 0.39$ & $0.73 \pm 0.34$ \\
\hline Hypertriglyceridemia (2) & $1.01 \pm 0.28$ & $20.1 \pm 0.6$ & $0.193 \pm 0.022 \ddagger$ & $0.075 \pm 0.018$ & $0.026 \pm 0.002$ & $0.048 \pm 0.005$ & $1.51 \pm 0.31$ & $0.97 \pm 0.07$ \\
\hline All subjects (24) & $1.11 \pm 0.25$ & $27.5 \pm 5.8$ & $0.084 \pm 0.050$ & $0.050 \pm 0.018$ & $0.018 \pm 0.007$ & $0.025 \pm 0.015$ & $1.35 \pm 0.46$ & $0.66 \pm 0.36$ \\
\hline
\end{tabular}

Probability that value differs from normal by one way analysis of variance and use of the Dunnett procedure (see Methods): $* P<0.05 ; \ddagger P<0.01$; $\$ 0.05<P<0.06$. 
TABLE IV

Subjects Studied by Groups: $M_{2}$ and $M_{3}$ under Various Assumptions (mean $\pm S D$ )

\begin{tabular}{|c|c|c|c|c|c|c|c|}
\hline \multirow[b]{2}{*}{ Group (no. of subjects) } & \multicolumn{2}{|c|}{ Minimum } & \multicolumn{2}{|c|}{ Intermediate } & \multicolumn{2}{|c|}{ Maximum } & \multirow{2}{*}{$\frac{\text { Intermediate }}{M_{2}+M_{3}}$} \\
\hline & $\mathbf{M}_{2}$ & $\mathbf{M}_{3}$ & $\mathbf{M}_{2}$ & $\mathbf{M}_{2}$ & $\mathbf{M}_{2}$ & Ms & \\
\hline & \multicolumn{2}{|c|}{$g$} & \multicolumn{2}{|c|}{ g } & \multicolumn{2}{|c|}{ g } & $\boldsymbol{g}$ \\
\hline Normal (8) & $24.8 \pm 11.2$ & $24.1 \pm 9.4$ & $31.9 \pm 13.3$ & $45.3 \pm 11.1$ & $38.9 \pm 15.6$ & $66.4 \pm 17.8$ & $77.1 \pm 10.2$ \\
\hline Hypercholesterolemia (6) & $19.8 \pm 10.1$ & $40.5 \pm 15.4 \S$ & $30.1 \pm 5.8$ & $67.8 \pm 14.0 \ddagger$ & $40.5 \pm 8.3$ & $95.2 \pm 19.4^{*}$ & $97.9 \pm 18.4$ \\
\hline Mixed hyperlipidemia (8) & $19.7 \pm 9.5$ & $41.8 \pm 13.3^{*}$ & $27.3 \pm 11.6$ & $70.2 \pm 11.5 \ddagger$ & $34.9 \pm 14.0$ & $98.5 \pm 18.4^{*}$ & $97.4 \pm 18.1$ \\
\hline Hypertriglyceridemia (2) & $7.8 \pm 0.7$ & $36.9 \pm 5.5$ & $9.8 \pm 1.2$ & $52.0 \pm 12.1$ & $11.9 \pm 1.6$ & $67.2 \pm 18.6$ & $61.8 \pm 13.2$ \\
\hline All subjects (24) & $20.4 \pm 10.4$ & $35.2 \pm 14.1$ & $28.1 \pm 11.7$ & $59.8 \pm 16.1$ & $35.7 \pm 14.4$ & $84.4 \pm 23.1$ & $87.8 \pm 19.2$ \\
\hline
\end{tabular}

Probability that value differs from normal by one way analysis of variance and use of the Dunnett procedure (see Methods):

${ }^{*} P<0.05 ; \ddagger P<0.01 ; \& 0.05<P<0.06$.

area) were used as indices of overall body size; the other two variables (percent of ideal body weight and excess weight) were used as indices of adiposity. PR was highly significantly correlated $(P<0.01)$ with all four body size variables. The correlations were much stronger, however, with the indices of overall body size than with those of adiposity. Thus, a correlation coefficient $(r)$ of 0.80 was observed for the correlation of PR with either surface area or total body weight, whereas $r$ values of 0.42 and 0.45 were observed for the correlation, respectively, of $\mathrm{PR}$ with percent of ideal weight or with excess weight. In these subjects with nearly ideal body weights, approximately $64 \%\left(0.80^{2}\right.$ $\times 100$ ) of the observed differences in cholesterol PR, in the range of from 0.71 to $1.47 \mathrm{~g} / \mathrm{day}$, could be accounted for by differences in total body size. Correlations between PR and adiposity disappeared after correcting for body size.

TABLE V

Analysis for Simple Linear Correlations between Model Parameters and Selected Variables in 24 Subjects

\begin{tabular}{|c|c|c|c|c|c|}
\hline \multirow{2}{*}{$\begin{array}{l}\text { Model } \\
\text { param- } \\
\text { eters\| }\end{array}$} & \multirow[b]{2}{*}{ Age } & \multirow{2}{*}{$\begin{array}{c}\text { Surface } \\
\text { area }\end{array}$} & \multirow{2}{*}{$\begin{array}{l}\text { Excess } \\
\text { weight }\end{array}$} & \multicolumn{2}{|c|}{ Serum } \\
\hline & & & & Cholesterol & Triglyceride \\
\hline & $y r$ & $m^{2}$ & kg & \multicolumn{2}{|c|}{$m g / d l$} \\
\hline PR & -0.40738 & $0.7984 \ddagger$ & $0.4525 *$ & -0.1291 & 0.3072 \\
\hline $\mathbf{M}_{1}$ & 0.0817 & $0.4309 *$ & $0.6117 \ddagger$ & $0.5326 \ddagger$ & -0.0831 \\
\hline$k_{12}$ & 0.3265 & -0.0625 & 0.2044 & 0.0277 & 0.3212 \\
\hline$k_{21}$ & $-0.5612 \ddagger$ & 0.2608 & -0.1255 & $-0.6209 \ddagger$ & 0.1139 \\
\hline$k_{18}$ & 0.0080 & 0.1851 & 0.2625 & -0.2711 & 0.1443 \\
\hline$k_{31}$ & 0.3232 & -0.0068 & 0.2520 & 0.0243 & 0.3627 \\
\hline $\mathbf{M}_{2} \min$ & $-0.4838 *$ & 0.2808 & -0.0777 & -0.1737 & -0.1646 \\
\hline $\mathrm{M}_{\mathbf{3}} \min$ & $0.5388 \ddagger$ & -0.0451 & $0.4814^{*}$ & $0.7037 \ddagger$ & 0.2819 \\
\hline $\mathbf{M}_{2}$ inter & -0.3467 & 0.2978 & 0.0520 & -0.0275 & -0.2127 \\
\hline $\mathbf{M}_{3}$ inter & 0.3879 & 0.0263 & $0.5419 \ddagger$ & $0.7471 \ddagger$ & 0.3722 \\
\hline$M_{2} \max$ & -0.2143 & 0.2814 & 0.1409 & 0.0808 & -0.2270 \\
\hline$M_{3} \max$ & 0.2117 & 0.0643 & $0.4616^{*}$ & $0.6118 \ddagger$ & 0.3467 \\
\hline
\end{tabular}

The correlation coefficients are listed, with statistically significant values shown as: $* P<0.05 ; \ddagger P<0.01,80.05<P<0.06$.

$\| \min =$ minimum $;$ inter $=$ intermediate $; \max =$ maximum.
Table $\mathrm{V}$ presents the correlations found between the parameters of the three-pool model and the major physiological variables; in this table, surface area is used as an index of total body size, and excess weight as an index of adiposity. The $\mathrm{PR}$ was not correlated with either the serum cholesterol or triglyceride concentration, but was almost significantly correlated negatively with age $(P<0.06)$. For the four rate constants of transfer between pools, significant correlations (negatively with age and with serum cholesterol level) were only found for $k_{21}$. $\mathrm{M}_{1}$ was correlated both with indices of adiposity and of body size, but most strongly with excess weight. $M_{1}$ was also correlated with the serum cholesterol level. All three estimates of $\mathrm{M}_{\mathrm{s}}$ (minimum, intermediate, and maximum) were significantly correlated with excess weight (but not with surface area), and with the serum cholesterol level. The minimum $\mathrm{M}_{3}$ was also significantly correlated with age.

To explore further the relationships between the physiological variables and the parameters of the threepool model, a multiple linear regression analysis was performed. The necessity for such analysis is evident from consideration of the results obtained with the PR. Thus, since body size is the major determinant of $P R$, it is clear that $P R$ values must be adjusted for body size differences before looking for other correlations. For each model parameter, the body size variable showing the best correlation with that parameter in the linear correlation analysis was used as an independent variable, together with the serum concentrations of cholesterol and triglyceride.

The results of this analysis are shown in Table VI. After adjusting for the effects of body size, the serum cholesterol level was found to be significantly correlated with the same model parameters $\left(M_{1}\right.$, all estimates of $\mathrm{M}_{3}$, intermediate $\mathrm{M}_{\mathbf{2}}+\mathrm{M}_{3}, k_{21}$ ) with which it had been found correlated in the simple linear regression analysis (Table V). On multiple regression analysis, however, the serum triglyceride level was found to be 
TABLE VI

Multiple Regression Analysis Relating Parameters of the Three-Pool Model to Body Size and Serum Lipid Levels in 24 Subjects

\begin{tabular}{|c|c|c|c|c|}
\hline \multirow[b]{3}{*}{$\begin{array}{l}\text { Dependent } \\
\text { variable } 8\end{array}$} & \multirow{2}{*}{\multicolumn{2}{|c|}{ Body size }} & \multicolumn{2}{|c|}{ Serum } \\
\hline & & & \multirow{2}{*}{$\begin{array}{l}\text { Choles- } \\
\text { terol } \\
\text { (F) }\end{array}$} & \multirow{2}{*}{$\begin{array}{l}\text { Tri- } \\
\text { glyc- } \\
\text { eride } \\
\text { (F) }\end{array}$} \\
\hline & $\begin{array}{l}\text { Vari- } \\
\text { ablell }\end{array}$ & $\mathbf{F}$ & & \\
\hline PR & SA & $38.71 \ddagger$ & 0.03 & $7.45^{*}$ \\
\hline $\mathrm{M}_{1} \uparrow$ & SA & $21.84 \ddagger$ & $8.71 \ddagger$ & $12.15 \ddagger$ \\
\hline$k_{12}$ & EW & 0.64 & 0.38 & 0.64 \\
\hline$k_{21}$ & EW & 0.00 & $13.80 \ddagger$ & $5.69^{*}$ \\
\hline$k_{13}$ & EW & 2.84 & 1.75 & 1.54 \\
\hline$k_{31}$ & EW & 1.08 & 0.61 & 3.33 \\
\hline $\mathbf{M}_{2} \min$ & SA & 1.89 & 0.04 & 0.63 \\
\hline $\mathrm{M}_{\mathbf{3}} \min$ & EW & $5.84^{*}$ & $21.58 \ddagger$ & 0.01 \\
\hline $\mathbf{M}_{2}$ inter & SA & 2.15 & 0.52 & 1.09 \\
\hline $\mathbf{M}_{\mathbf{3}}$ inter & EW & $10.48 \ddagger$ & $27.78 \ddagger$ & 0.55 \\
\hline $\mathbf{M}_{2} \max$ & SA & 1.90 & 1.85 & 1.24 \\
\hline$M_{3} \max$ & EW & $4.50^{*}$ & $13.16 \ddagger$ & 0.54 \\
\hline $\mathbf{M}_{2}+\mathbf{M}_{3}$ inter & $\mathrm{EW}$ & $5.35^{*}$ & $12.96 \ddagger$ & 0.15 \\
\hline
\end{tabular}

The table presents the F-test values for each correlation, with significant values identified as: ${ }^{*} P<0.05, \ddagger P<0.01$.

$\delta \min =$ minimum; inter $=$ intermediate $; \max =$ maximum. $\| \mathrm{SA}=$ surface area $\mathrm{EW}=$ excess weight. In general, each dependent variable was analyzed with the body size variable with which it was best correlated (see text). For consistency, all intercompartmental rate constants $(k)$ used excess weight as the variable, and all estimates of $M_{2}$ used surface area.

If For $M_{1}$, when excess weight was used as the body size variable the F-test values for excess weight, cholesterol, and triglyceride were $13.15 \ddagger, 7.47^{*}$, and $5.88^{*}$, respectively.

significantly correlated with three model parameters ( $\mathrm{PR}, \mathrm{M}_{1}$, and $k_{21}$ ), whereas no significant correlations with triglyceride had been found by simple linear regression analysis.

Fig. 2 shows the relationships between the residual PR (that portion of the PR not accounted for after adjustment of the total PR for the effects of body size variation) and the concentration of serum triglyceride. 22 of the 24 triglyceride concentrations fell between 80 and $300 \mathrm{mg} / \mathrm{dl}$, while two patients had much higher concentrations, of 464 and $691 \mathrm{mg} / \mathrm{dl}$. When the entire population was analyzed as if homogeneous, the $r$ was $0.55(P<0.05)$ (solid regression line in Fig. 2). When the population of 22 subjects with triglyceride levels from 80 to 300 was considered as a group, however, no significant correlation was found between the residual $P R$ and the serum triglyceride level. Neither the slope of the regression line for this population (dashed line in Fig. 2) nor its $r$ value (0.19) were significant.

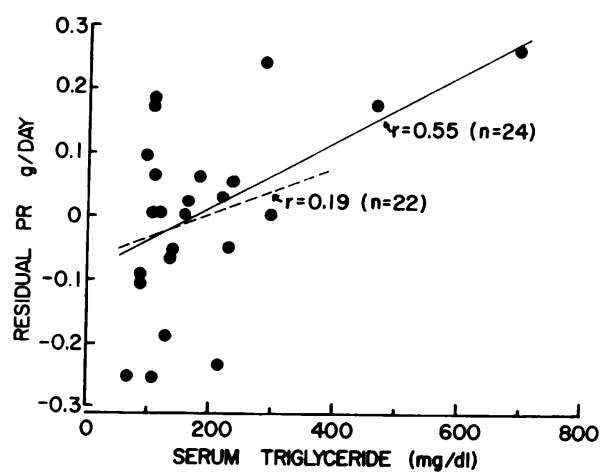

FIGURE 2 Relationships between the residual PR of cholesterol (that portion of the PR not accounted for after adjustment for the effects of body size variation) and the concentration of serum triglyceride.

Fig. 3 shows the relationship between the minimum value of the size of pool 3 (minimum $\mathrm{M}_{s}$ ) and the concentration of serum cholesterol. The values of Ms shown in this figure were adjusted for the effects of excess weight (i.e. adjusted to the equivalent of zero excess weight) by multiple regression analysis. The partial correlation coefficient for the data shown in Fig. 3 is 0.70 . The multiple regression equation relating minimum $\mathrm{Ms}$ to excess weight (EW) and serum cholesterol level $(\mathrm{CL})$ is :

$$
\text { minimum } \mathrm{M}_{\mathrm{s}}=7.80+0.8122 \cdot \mathrm{EW}+0.08036 \cdot \mathrm{CL} \text {. }
$$

Since the values of $k_{21}$ and of minimum $\mathrm{M}_{\mathbf{s}}$ were significantly correlated with age with $P<0.01$ (Table $\mathrm{V})$, an additional multiple regression analysis was carried out for these model parameters (and for the other estimates of $\mathrm{M}_{\mathbf{s}}$ as well) in which four independent variables (excess weight, age, cholesterol, and triglyceride levels) were tested. The results of this analysis

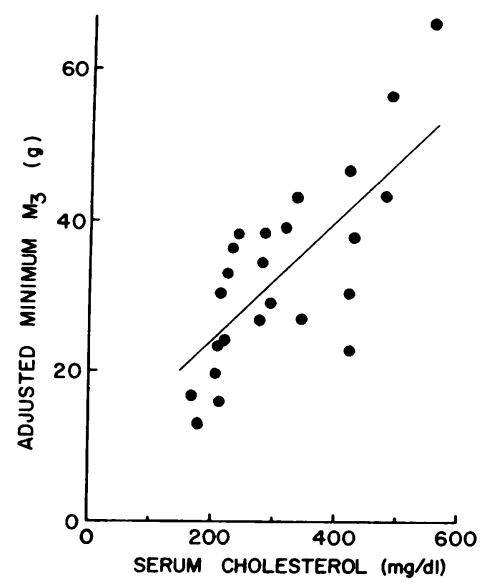

Figure 3 Relationship between minimum $M_{3}$, adjusted for the effects of excess weight, and the concentration of serum cholesterol. 
confirmed the results shown in Table VI. In this additional analysis (with age included as another independent variable) all three estimates of $\mathrm{M}_{3}$ were still found significantly correlated with the serum cholesterol level $(P<0.01)$ and with excess weight $(P<$ 0.01 for intermediate $\mathrm{M}_{3}$ and $P<0.05$ for minimum and maximum $\mathrm{M}_{3}$ ). Only minimum $\mathrm{M}_{3}$ was correlated $(P<0.05)$ with age. $k_{21}$ was still found significantly correlated with both serum cholesterol $(P<0.01)$ and triglyceride $(P<0.05)$ levels, but not with age or with excess weight.

Relationship between $P R$ values from long-term and shorter studies. Calculations were carried out to compare the values for PR obtained by three-pool model analysis of these long-term studies with the values of PR which would have been obtained in short-term studies. Each of the 24 sets of long-term data was truncated at $12 \mathrm{wk}$, and the PR calculated by two-pool model analysis of the resulting data $(1,2,7)$. As has been reported previously (6) for only six subjects, the short-term studies underestimate the area under the plasma turnover curve, and thus overestimate the PR. The mean PR from the 12 wk of data was $1.213 \pm 0.256$ (SD), whereas the PR from the full long-term data was $1.105 \pm 0.245 \mathrm{~g} /$ day. The difference between the $P R$ as calculated from the long-term data and from the 12 wk data was $9.0 \pm 2.9 \%$ (mean $\pm \mathrm{SD}$ ) (of the 12-wk value), with a range in percent differences of 3.9$14.6 \%$. The relationship between the PR from longterm data $\left(\mathrm{PR}_{\mathbf{L T}}\right)$ and the $\mathrm{PR}$ from $12 \mathrm{wk}$ data $\left(\mathrm{PR}_{12}\right)$ was :

$$
\mathrm{PR}_{\mathrm{LT}}=-0.043+0.9467 \mathrm{PR}_{\mathbf{1 a}} ; r=0.989 \text {. }
$$

The slope of this regression line is significant $(P<$ 0.01 ), but the intercept is not. In contrast to $P R$, as reported previously $M_{1}$ calculated from the truncated data was not significantly different from $\mathbf{M}_{\mathbf{1}}$ calculated with the full long-term data, since with either shortterm or long-term data the zero time intercept is well determined.

\section{DISCUSSION}

The aim of these studies was to determine first whether the same compartmental model would be appropriate for the description of the long-term turnover of plasma cholesterol in both normal subjects and in patients with different kinds of hyperlipidemia. If the same model were appropriate, the studies then aimed to compare normal and hyperlipidemic persons with regard to each of the parameters of the model.

We have previously reported (6) that, in each of three normal and three hypertriglyceridemic subjects, a three-pool model provided a significantly better description of the long-term turnover curve than did a two-pool model. The results reported here extend this series to a total of 24 subjects, including 8 normal controls, 14 patients with hypercholesterolemia (8 with concurrent hypertriglyceridemia), and 2 patients with hypertriglyceridemia alone. Many of the hyperlipidemic patients had a definite familial disorder; in some of the patients familial disease was not present. Despite the heterogeneous characteristics of the hyperlipidemic population studied, however, the same three-compartment model provided the best fit to the long-term turnover data in each of the 23 subjects in whom complete data were collected. Although the population studied is still not large, these findings suggest that the threepool model may well be generally valid for the study of cholesterol turnover in humans.

This conclusion is somewhat at variance with that of Samuel and Lieberman (21), who conducted plasma cholesterol turnover studies on 17 patients for intervals longer than $50 \mathrm{wk}$, and reported that in 12 patients a three-exponential curve fit was better, whereas in 5 patients two-exponential fits were better. The data were, accordingly, analyzed by "input-output analysis" $(3,5)$, which is independent of the number of exponentials, but which limits the amount of information which can be derived from the data as compared with that obtainable by multicompartmental analysis. We believe that there are significant differences between the methods of curve fitting and data analysis employed by Samuel and Lieberman and by ourselves, and that these differences could in part account for the finding of a two-exponential, rather than a three-exponential fit in five patients by these investigators. Additional studies will be required to fully resolve this question.

The data reported here were analyzed first by dividing the patients into groups according to the kind of lipid elevation present, and comparing the values for the model parameters between groups. Single and multiple regression analyses were then conducted to explore possible correlations between the model parameters and various physiological variables.

Using this approach, significant differences between groups, or correlations with serum lipid levels were seen for several parameters of the three-pool model: PR, $k_{21}$, $M_{1}$, and all estimates of $M_{3}$. Many of the model parameters, however, showed no differences between hyperlipidemic and normal subjects, and no correlations with serum lipid concentrations. These model parameters, which were unaffected by hyperlipidemia or lipid levels, included $k_{13}, k_{81}$, and all estimates of $\mathrm{M}_{2}$. In addition, although $k_{12}$ was elevated in the two hypertriglyceridemic patients studied, the absence of a significant correlation between $k_{12}$ and either serum cholesterol or triglyceride concentration, by single or multiple regression analysis, suggests that when a larger number of hyper- 
triglyceridemic patients are studied this parameter may well be found to be unrelated to serum lipid levels.

The studies reported here also permitted us to extend and make more firm the relationship between the $\mathrm{PR}$ as calculated from a 12 -wk study (with the twopool model) and that calculated from a long-term study (with the three-pool model). In these 24 studies, the difference between the PR as calculated from the longterm data and from 12 wk of data was $9.0 \pm 2.9 \%$ (mean $\pm \mathrm{SD})$. This confirms our conclusion (6) that previous estimates of the $\mathrm{PR}$ obtained from mediumterm studies can be considered as quantitatively valid if corrected by a reduction of $9 \%$. With such a correction, the $\mathrm{PR}$ as calculated from medium-term kinetic studies agrees extremely closely with the total body turnover rate as measured directly by sterol balance methods (4, 22).

Previous studies of cholesterol turnover, using either the two-pool model (2) or input-output analysis (21), have demonstrated very similar turnover rates of cholesterol in patients with hypercholesterolemia and in normal subjects. In the present study differences in production rates were not seen between hypercholesterolemic patients and normals, nor was the PR correlated with the serum cholesterol level. It was previously suggested (2) that hypercholesterolemia is not associated with abnormal rates of formation or excretion of cholesterol in the body, but with some impediment to the "clearance" or excretion of cholesterol from the plasma pool. This conclusion is consistent with the finding of a decreased fractional rate of low density lipoprotein (LDL) degradation in patients with familial hypercholesterolemia (23). Insight into the mechanism responsible for the decreased fractional rate of LDL degradation has been provided by recent studies with fibroblasts from patients with familial hypercholesterolemia (24). These fibroblasts have a reduced number of specific binding sites for LDL, and show a diminished catabolism of LDL in culture in vitro.

The effect of hypertriglyceridemia on the turnover rate of cholesterol is not comparably clear. Sodhi and Kudchodkar reported that patients with hypercholesterolemia and hypertriglyceridemia had markedly higher cholesterol turnover rates than did patients with hypercholesterolemia alone, as determined by both sterol balance and kinetic (two-pool model) studies (25). In contrast, using sterol balance methods, Miettinen did not find an increased daily turnover rate of cholesterol in patients with hypertriglyceridemia, particularly when the data were corrected for differences in obesity and body weight (26). More recently, Grundy has reported that sterol balance studies in his laboratory do not support the claim that the majority of patients with hypertriglyceridemia have increased rates of sterol syn- thesis (turnover) (27). In the present study, patients with hypertriglyceridemia (either with or without hypercholesterolemia) had PRs which were not significantly different from those of normal subjects. In addition, the PR did not correlate with the serum triglyceride concentration on simple linear regression analysis. After adjusting for the effects of body size by multiple regression analysis, however, a probably significant correlation $(P<0.05)$ was found between the $\mathrm{PR}$ and the serum triglyceride level. Further examination of the data showed that this correlation was dependent upon the results found with the two patients with markedly elevated triglyceride levels (of 464 and 691). Thus, when the population of 22 subjects with triglyceride levels from 80 to 300 was analyzed as a group, no significant correlation was found between the residual $\mathrm{PR}$ and the triglyceride level (Fig. 2). Our interpretation of these data is that patients with extremely high triglyceride levels may represent a different population, with regard to the regulation of cholesterol turnover, from those with normal or moderately elevated triglyceride levels. This conclusion is consonant with Grundy's suggestion (27) that a subgroup of hypertriglyceridemic patients may well exist who show an increased synthesis of cholesterol, although this is not found in most patients with hypertriglyceridemia.

Since bile acids represent the major catabolic pathway in cholesterol metabolism, bile acid metabolism in hyperlipidemia has been studied by several investigators. Somewhat discordant results have been reported by different laboratories. A decreased fecal excretion rate of bile acids has been reported for patients with familial hypercholesterolemia, with a low normal rate seen in other patients with "essential hypercholesterolemia" (28). More recently, patients with hypercholesterolemia and the type II pattern were reported to have total bile acid formation within normal limits, but with a diminished synthesis of cholic acid seen in patients with the type IIa pattern (29). The bile acid turnover or excretion in patients with hypercholesterolemia and hypertriglyceridemia has been reported to be much higher than that in patients with hypercholesterolemia alone $(30,31)$, although this has not been found in all studies $(28,29)$. Bile acid turnover or excretion have been reported to be considerably increased in patients with hypertriglyceridemia alone $(29,32)$ although this too has not been found in all studies (27). As with cholesterol turnover (see above) it is probable that hypertriglyceridemic patients represent a heterogeneous population with regard to bile acid metabolism, and that there are subsets of such patients who manifest increased synthesis and turnover of bile acids, but that this is not a universal phenomenon. 
In the series reported here, the major determinant of cholesterol production rate was overall body size, expressed either as total body weight or as surface area. Thus, differences in total body size accounted for $64 \%$ of the observed differences in cholesterol PR. For this entire series the mean $( \pm S D) P R$ was $15.2 \pm 2.0 \mathrm{mg} / \mathrm{kg}$ body wt per day. The corresponding values for the normal, hypercholesterolemic, and mixed hyperlipidemic groups were $14.9 \pm 2.1,14.9 \pm 2.2$, and $16.0 \pm 2.0 \mathrm{mg} / \mathrm{kg}$ per day, respectively.

Total weight and excess weight have been reported previously to influence cholesterol $\operatorname{PR}(2,26,33)$. Obese subjects have been shown to have increased cholesterol PR both by sterol balance techniques (26, $33)$, and by isotope kinetic analysis with the two-pool model $(2,33)$. In the morbidly obese patients (mean percent ideal body weight 257) studied by Nestel et al. (33), cholesterol synthesis rates were highly correlated with both excess body weight and with adipose cellularity, with correlation coefficients of 0.66 and 0.72 , respectively. Analysis of the data reported by these workers reveals that an even slightly stronger correlation $(r=0.73)$ exists between total body weight and cholesterol synthesis rate in the eight patients studied. In the patients reported here, the maximum excess body weight was $20 \mathrm{~kg}$ while the minimum excess weight in the patients reported by Nestel et al. (33) was $43 \mathrm{~kg}$, so that there is no overlap in the weight characteristics of the two study populations. In our group of nonobese subjects, cholesterol PR was much more highly correlated with indices of total body size than with indices of adiposity. In morbid obesity, as the degree of adiposity increases relative to body surface area, the adipose organ appears to play an increasingly significant role in influencing total body cholesterol production. This role can, however, be considered at least as well in terms of total weight rather than excess weight. Moreover, recent studies (34) have indicated that adipose tissue cholesterol synthesis itself contributes very little, if at all, to the enhanced cholesterol synthesis of obesity.

In our study population, $M_{1}$, the size of the rapidly exchangeable compartment, was found to be significantly correlated with all four variables of body size (weight, surface area, percent of ideal weight, and excess weight), but most strongly with excess weight. When the data were adjusted for the effects of body size, significant correlations were found between $M_{1}$ and both the serum concentration of cholesterol and that of triglyceride. Although the quantitative effects of serum lipid elevations on $\mathrm{M}_{1}$ are small, they are statistically highly significant by multiple regression analysis. Previous kinetic studies have shown increases in the size of the rapidly miscible pool in patients with hypercholesterolemia (2), and this has been interpreted as reflecting the increased cholesterol content of the plasma compartment in such patients (2). A similar interpretation cannot be proposed for the present new finding of a relationship between serum triglyceride level and $M_{1}$, which remains to be explained.

Compartmental analysis with the three-pool model demonstrated major differences in the relationships between the physiological variables and the sizes of the two more slowly exchanging compartments, pools 2 and 3. The size of pool $2\left(\mathrm{M}_{2}\right)$, the pool which consists of cholesterol which equilibrates at an intermediate rate with plasma cholesterol, was correlated neither with any of the indices of body size or adiposity, nor with the levels of either serum cholesterol or triglyceride. In contrast, all estimates of $\mathrm{Ms}$, the size of the most slowly turning over pool 3 , were significantly correlated with indices of adiposity (both percent of ideal weight and excess weight) and with the serum cholesterol concentration. The positive correlation between $\mathrm{M}_{8}$ and adiposity is consistent with the finding that adipose tissue cholesterol appears to be an important part of the most slowly turning over compartment, pool 3 (34). Schreibman and Dell have reported recently cholesterol turnover studies of 10-20 wk duration in which a threepool model was fit simultaneously to both plasma and adipocyte specific activity-time curves (34). In five of six subjects, the kinetics of adipose tissue cholesterol closely fit that of the slowly turning over pool 3 . Cholesterol in other peripheral tissues, particularly connective tissue and skeletal muscle (35-37), and including arterial walls (38), also equilibrates slowly with plasma cholesterol; cholesterol in these tissue sites probably also comprises a significant portion of pool 3 . The findings reported here suggest that the amounts of cholesterol in these slowly equilibrating tissue sites increase in relation to elevations in the serum cholesterol level. The increased accumulation of cholesterol in atherosclerotic arteries in patients with hypercholesterolemia may represent one aspect of this phenomenon.

The observed correlation between the size of pool 3 $\left(\mathrm{M}_{3}\right)$ and the serum cholesterol concentration suggests that the kinetic analysis used here can provide a method for estimating the size of pathological accumulations of cholesterol in slowly equilibrating tissue sites. If this is true, then it may be possible to study the effects of therapeutic intervention (e.g. lipid lowering drugs) on the amount of cholesterol in tissue pools, by repeating long-term studies of cholesterol turnover after a period of therapy in specific patients.

In addition to its relationships with excess weight and serum cholesterol level, the minimum value of $\mathrm{M}_{\mathbf{s}}$ was also significantly $(P<0.01)$ correlated with age. Although the other estimates of $M_{8}$ (intermediate and 
maximum $\mathrm{M}_{3}$ ) showed positive correlation coefficients with age, the values were not significant statistically. Since, as previously pointed out (6), it is probable that the true values for $\mathrm{M}_{3}$ (and $\mathrm{M}_{2}$ ) are much closer to the lower than the upper limiting values (34), the observed correlation of minimum $\mathrm{Ms}_{\mathbf{s}}$ with age can be considered to support the observations of Crouse et al. (39) showing an increase in cholesterol concentration in connective tissue with age. The observed correlation (and the observed negative correlation between minimum $\mathrm{M}_{2}$ and age) may also reflect the changes in body composition, particularly the relative increase in the amount of fat tissue in the body, which occur with increasing age.

\section{ACKNOWLEDGMENTS}

We thank Ms. M. Myers, Ms. B. Adams, Ms. M. Alvir, and Mr. R. Sciacca for expert assistance.

This work was supported by grants HL-14236 (SCR) and AM-05968 from the National Institutes of Health, and by grants from the Sharon Research Institute and the William Lightfoot Schultz Foundation.

\section{REFERENCES}

1. Goodman, DeW. S., and R. P. Noble. 1968. Turnover of plasma cholesterol in man. J. Clin. Invest. 47: 231241 .

2. Nestel, P. J., H. M. Whyte, and DeW. S. Goodman. 1969. Distribution and turnover of cholesterol in humans. J. Clin. Invest. 48: 982-991.

3. Perl, W., and P. Samuel. 1969. Input-output analysis for total input rate and total traced mass of body cholesterol in man. Circ. Res. 25: 191-199.

4. Grundy, S. M., and E. H. Ahrens, Jr. 1969. Measurements of cholesterol turnover, synthesis, and absorption in man, carried out by isotopic kinetic and sterol balance methods. J. Lipid Res. 10: 91-107.

5. Samuel, P., and W. Perl. 1970. Long-term decay of serum cholesterol radioactivity: body cholesterol metabolism in normals and in patients with hyperlipoproteinemia and atherosclerosis. J. Clin. Invest. 49: 346357.

6. Goodman, DeW. S., R. P. Noble, and R. B. Dell. 1973. Three-pool model of the long-term turnover of plasma cholesterol in man. J. Lipid Res. 14: 178-188.

7. Goodman, DeW. S., R. P. Noble, and R. B. Dell. 1973. The effects of colestipol resin and of colestipol plus clofibrate on the turnover of plasma cholesterol in man. J. Clin. Invest. 52 : 2646-2655.

8. Fredrickson, D. S., R. I. Levy, and R. S. Lees. 1967. Fat transport in lipoproteins-an integrated approach to mechanisms and disorders. N. Engl. J. Med. 276: 34-44, 94-103, 148-156, 215-225, 273-281.

9. Beaumont, J. L., L. A. Carlson, G. R. Cooper, Z. Fejfar, D. S. Fredrickson, and T. Strasser. 1970. Classification of hyperlipidaemias and hyperlipoproteinaemias. Bull. W. H. O. 43: 891-908.

10. Noble, R. P. 1968. Electrophoretic separation of plasma lipoproteins in agarose gel. J. Lipid Res. 9: 693-700.

11. Goldstein, J. L., H. G. Schrott, W. R. Hazzard, E. L. Bierman, and A. G. Motulsky. 1973. Hyperlipidemia in coronary heart disease. II. Genetic analysis of lipid levels in 176 families and delineation of a new inherited disorder, combined hyperlipidemia. J. Clin. In vest. 52: 1544-1568.

12. Goldstein, J. L., W. R. Hazzard, H. G. Schrott, E. L. Bierman, and A. G. Motulsky. 1973. Hyperlipidemia in coronary heart disease. I. Lipid levels in 500 survivors of myocardial infarction. J. Clin. Invest. 52: 1533-1543.

13. Block, W. D., K. J. Jarret, and J. B. Levine. 1965. Use of a single color reagent to improve the automated determination of serum total cholesterol. In Automation in Analytical Chemistry. L. T. Skeggs, Jr., editor. Mediad, New York. 345-347.

14. Noble, R. P., and F. M. Campbell. 1970. Improved accuracy in automated fluorometric determination of plasma triglycerides. Clin. Chem. 16: 166-170.

15. Kessler, G., and H. Lederer. 1965. Fluorometric measurements of triglycerides. In Automation in Analytical Chemistry. L. T. Skeggs, Jr., editor. Mediad, New York. 341-344.

16. Dell, R. B., R. Sciacca, K. Lieberman, D. B. Case, and P. J. Cannon. 1973. A weighted least-squares technique for the analysis of kinetic data and its application to the study of renal ${ }^{133}$ Xenon washout in dogs and man. Circ. Res. 32: 71-84.

17. Du Bois, D., and E. F. Du Bois. 1916. Clinical calorimetry. X. A formula to estimate the approximate surface area if height and weight be known. Arch. Intern. Med. 17 : 863-871.

18. Metropolitan Life Insurance Co. 1959. Statistical Bulletin 40.

19. Steel, R. G. D., and J. H. Torrie. 1960. Principles and Procedures of Statistics. McGraw-Hill Book Co., New York. 99-131.

20. Draper, R. N., and H. Smith. 1966. Applied Regression Analysis. John Wiley and Sons, New York.

21. Samuel, P., and S. Lieberman. 1973. Improved estimation of body masses and turnover of cholesterol by computerized input-output analysis. J. Lipid Res. 14: 189196.

22. Salen, G., E. H. Ahrens, Jr., and S. M. Grundy. 1970. Metabolism of $\beta$-sitosterol in man. J. Clin. Invest. 49: 952-967.

23. Langer, T., W. Strober, and R. I. Levy. 1972. The metabolism of low density lipoprotein in familial type II hyperlipoproteinemia. J. Clin. Invest. 51: 1528-1536.

24. Goldstein, J. L., and M. S. Brown. 1974. Binding and degradation of low density lipoproteins by cultured human fibroblasts. Comparison of cells from a normal subject and from a patient with homozygous familial hypercholesterolemia. J. Biol. Chem. 249: 5153-5162.

25. Sodhi, H. S., and B. J. Kudchodkar. 1973. Synthesis of cholesterol in hypercholesterolemia and its relationship to plasma triglycerides. Metab. (Clin. Exp.). 22: 895912.

26. Miettinen, T. A. 1971. Cholesterol production in obesity. Circulation. 44 : 842-850.

27. Grundy, S. M. 1975. Effects of polyunsaturated fats on lipid metabolism in patients with hypertriglyceridemia. J. Clin. Invest. 55 : 269-282.

28. Miettinen, T. A. 1973. Clinical implications of bile acid metabolism in man. In The Bile Acids: Chemistry, Physiology and Metabolism. Volume 2. Physiology and Metabolism. P. P. Nair and D. Kritchevsky, editors. Plenum Publishing Corporation, New York. 191-247.

29. Einarsson, K., K. Hellström, and M. Kallner. 1974. Bile acid kinetics in relation to sex, serum lipids, body 
weights, and gallbladder disease in patients with various types of hyperlipoproteinemia. J. Clin. Invest. 54 : 13011311.

30. Kottke, B. A. 1969. Differences in bile acid excretion. Primary hypercholesteremia compared to combined hypercholesteremia and hypertriglyceridemia. Circulation. 40: $13-20$.

31. Sodhi, H. S., and B. J. Kudchodkar. 1973. Catabolism of cholesterol in hypercholesterolemia and its relationship to plasma triglycerides. Clin. Chim. Acta. 46: 161171.

32. Nestel, P. J., and J. D. Hunter. 1974. Differences in bile acid excretion in subjects with hypercholesterolaemia, hypertriglyceridaemia and overweight. Aust. N. Z. J. Med. 4 : 491-496.

33. Nestel, P. J., P. H. Schreibman, and E. H. Ahrens, Jr. 1973. Cholesterol metabolism in human obesity. $J$. Clin. Invest. 52 : 2389-2397.

34. Schreibman, P. H., and R. B. Dell. 1975. Human adi- pocyte cholesterol. Concentration, localization, synthesis, and turnover. J. Clin. Invest. 55: 986-993.

35. Field, H., Jr., L. Swell, P. E. Schools, Jr., and C. R. Treadwell. 1960. Dynamic aspects of cholesterol metabolism in different areas of the aorta and other tissues in man and their relationship to atherosclerosis. Circulation. 22: 547-558.

36. Chobanian, A. V., and W. Hollander. 1962. Body cholesterol metabolism in man. I. The equilibration of serum and tissue cholesterol. J. Clin. Invest. 41: 1732-1737.

37. Wilson, J. D. 1970. The measurement of the exchangeable pools of cholesterol in the baboon. J. Clin. Invest. 49: 655-665.

38. Jagannathan, S. N., W. E. Connor, W. H. Baker, and A. K. Bhattacharyya. 1974. The turnover of cholesterol in human atherosclerotic arteries. J. Clin. Invest. 54: 366-377.

39. Crouse, J. R., S. M. Grundy, and E. H. Ahrens, Jr. 1972. Cholesterol distribution in the bulk tissues of man: variation with age. J. Clin. Invest. 51: 1292-1296. 